\title{
MicroRNA promoter methylation: a new tool for accurate detection of urothelial carcinoma
}

\author{
Nuno André Padrão ${ }^{1}$, Sara Monteiro-Reis ${ }^{1}$, Jorge Torres-Ferreira ${ }^{1,2}$, Luís Antunes ${ }^{3}$, Luís Leça ${ }^{2}$, Diana \\ Montezuma $^{1,2}$, João Ramalho-Carvalho ${ }^{1}$, Paula C Dias ${ }^{1,2}$, Paula Monteiro ${ }^{1,2}$, Jorge Oliveira ${ }^{4}$, Rui Henrique ${ }^{1,2,5}$ \\ and Carmen Jerónimo*,1,5 \\ ${ }^{1}$ Cancer Biology and Epigenetics Group - Research Center (CIIPOP), Portuguese Oncology Institute of Porto (IPO Porto), Rua Dr \\ António Bernardino de Almeida, Porto 4200-072, Portugal; ${ }^{2}$ Department of Pathology, Portuguese Oncology Institute of Porto \\ (IPO Porto), Rua Dr António Bernardino de Almeida, Porto 4200-072, Portugal; ${ }^{3}$ Department of Epidemiology, Portuguese \\ Oncology Institute of Porto (IPO Porto), Rua Dr António Bernardino de Almeida, Porto 4200-072, Portugal; ${ }^{4}$ Department of \\ Urology, Portuguese Oncology Institute of Porto (IPO Porto), Rua Dr António Bernardino de Almeida, Porto 4200-072, Portugal \\ and ${ }^{5}$ Department of Pathology and Molecular Immunology, Institute of Biomedical Sciences Abel Salazar (ICBAS) - University of \\ Porto, Rua de Jorge Viterbo Ferreira n. ${ }^{\circ} 228$, Porto 4050-313, Portugal
}

Background: Urothelial carcinoma (UC) is the most common cancer affecting the urinary system, worldwide. Lack of accurate early detection tools entails delayed diagnosis, precluding more efficient and timely treatment. In a previous study, we found that miR129-2 and miR-663a were differentially methylated in UC compared with other genitourinary tract malignancies. Here, we evaluated the diagnostic performance of those microRNAs in urine.

Methods: Promoter methylation levels of miR-129-2 and miR-663a were assessed, using real-time quantitative methylationspecific PCR, in UC tissue samples (using normal urothelium as control) and, subsequently, in urine samples from UC and other genitourinary malignancies. Diagnostic and prognostic performances were evaluated by receiver operator characteristics curve and survival analyses, respectively.

Results: Promoter methylation levels of both microRNAs were significantly higher in UC tissue samples compared with normal urothelium. In urine, the assay was able to distinguish UC from other genitourinary tract carcinomas with $87.7 \%$ sensitivity and $84 \%$ specificity, resulting in $85.85 \%$ overall accuracy.

Conclusions: This panel of miRNAs promoter methylation accurately detects UC in urine, comparing well with other promising epigenetic-based biomarkers. This may constitute the basis for a non-invasive assay to detect UC.

Urothelial carcinoma (UC), which affects the upper (renal pelvis and ureters) and lower (bladder, urethra) urinary tract, is the fourth most common cancer type in worldwide males, with 330380 new cases diagnosed in 2012, mostly afflicting elderly individuals (Torre et al, 2015). Haematuria is the most common clinical sign of UC, particularly of those arising in the lower urinary tract, but several prevalent benign conditions, such as urinary tract infection and/or lythiasis, are also associated with haematuria, thus limiting its cancer specificity. Moreover, upper tract UC (UTUC), although much less common (5-10\% of all cases), is mostly clinically asymptomatic. Consequently, although upper and lower tract UC display clinical and genomic similarities (Zhang et al, 2010), 60\% of UTUC are diagnosed at invasive stage, contrasting with $10 \%$ of bladder UC (BUC; Margulis et al, 2009). Thus, early detection is decisive to improve patient's survival.

Currently, BUC diagnosis usually consists on non-invasive (voided) urine cytology (which displays modest accuracy), followed by cytoscopic examination (Kaufman et al, 2009), whereas

*Correspondence: Dr C Jerónimo; E-mail: carmenjeronimo@ipoporto.min-saude.pt

Received 12 August 2016; revised 14 November 2016; accepted 20 December 2016; published online 12 January 2017

(c) 2017 Cancer Research UK. All rights reserved 0007-0920/17 
suspected cases of UTUC are investigated with computer tomographic urography or urinary cytology followed by ureteroscopy, but these methods have low sensitivity, especially for lowgrade tumours, and are often associated with patient discomfort (Kaufman et al, 2009; Remzi et al, 2011; Rouprêt et al, 2011). Follow-up of patients with UC is also based on periodic cystoscopy, an invasive, uncomfortable and expensive procedure, making UC one of the heaviest economical burdens in health systems (Lokeshwar et al, 2005). Thus, early, accurate and non-invasive diagnostic tools are critical to improve patient outcome and increase the cost-effectiveness of follow-up procedures.

MicroRNAs (miRNAs) are small ( $\sim 22$ nucleotides in length), non-coding RNA molecules involved in many important regulatory pathways including cell grow, proliferation, differentiation and cell death (Bartel, 2009; Silahtaroglu and Stenvang, 2010). In animals, they regulate the expression of complementary mRNA, thus inhibiting protein expression (Ambros, 2004). Recently, the role of deregulated miRNAs in oncogenesis has been emphasised and depending on its function and type of abnormal expression, they might act as oncogenes or tumour suppressor genes, in many types of cancer (Volinia et al, 2006). Expression of miRNAs might be epigenetically regulated, namely through methylation of $\mathrm{CpG}$ islands located at promoter regions, as well as histone posttranslational modifications. Alterations in those mechanisms might deregulate miRNAs expression in cancer cells and might, thus, be used advantageously as specific cancer biomarkers early detection, diagnosis, prognostication, prediction of response to treatment and monitoring (Silahtaroglu and Stenvang, 2010).

In search for epigenetic biomarkers in genitourinary cancer, we identified two miRNAs - miR-129-2 and miR-663a - that displayed significantly higher promoter methylation levels in a small series of BUC tissues. Thus, we aimed at validating that finding in larger series of UC, encompassing BUC and UTUC tissues, and test the feasibility of using miR-129-2 and miR-663a quantitative promoter methylation as a tool for accurate noninvasive detection of UC in voided urine, emphasising its specificity for UC among genitourinary malignancies.

\section{MATERIALS AND METHODS}

Patients and tumour sample collection. One hundred and fourteen BUC tissue samples were obtained from a consecutive series of patients diagnosed and treated with transurethral resection or radical cystectomy, with no previous history of UTUC, between 2005 and 2014, and 55 UTUC samples were obtained from another consecutive series of patients diagnosed and treated with radical nephroureterectomy or ureterectomy, with no previous history of BUC, between 2000 and 2011. Both the groups of patients were followed-up at the Portuguese Oncology Institute of Porto, Portugal. For the BUC samples, a small tumour sample was immediately snap-frozen, stored at $-80^{\circ} \mathrm{C}$ and subsequently cut in cryostat for DNA extraction. Routine collection and processing of tissue sample allowed for pathological examination, classification, grading and staging (Eble et al, 2004; Edge et al, 2010). UTUC samples were obtained from routinely-fixed and paraffin-embedded tissue used for pathological assessment (Eble et al, 2004; Edge et al, 2010). Controls for BUC consisted on an independent set of 19 normal bladder mucosae collected from $\mathrm{BlCa}$-free individuals (prostate cancer patients submitted to radical prostatectomy), and 31 paraffin-embedded normal upper tract urothelium (NUTU) set of samples obtained from renal cell carcinoma patients were used as UTUC controls. Relevant clinical data were collected from clinical charts and is depicted in Table 1.

Urine sample collection and processing. Voided urine (one sample per patient) was collected from 49 patients with BUC and
Table 1. Clinical and histopathological characteristics of patients with urothelial carcinoma and providers of normal urothelium

\begin{tabular}{|l|c|c|c|}
\hline Clinicopathological features & UC & $\begin{array}{c}\text { Normal } \\
\text { urothelium }\end{array}$ & P-value \\
\hline Patients, $n$ & 169 & 50 & \\
\hline $\begin{array}{l}\text { Gender, } n(\%) \\
\text { Male }\end{array}$ & $130(77 \%)$ & $38(76 \%)$ & \\
Female & $39(23 \%)$ & $12(24 \%)$ & \\
\hline Median age, years (range) & $73(42-93)$ & $62.5(48-82)$ & $P<0.001$ \\
\hline Pathological stage, $n(\%)$ & & & \\
PTa & $43(26 \%)$ & NA & \\
PT1 & $63(37 \%)$ & NA & \\
PT2 & $31(18 \%)$ & NA & \\
PT3 & $25(15 \%)$ & NA & \\
PT4 & $7(4 \%)$ & NA & \\
\hline Grade, $n$ (\%) & & & \\
Papillary, low grade & $59(35 \%)$ & NA & \\
Papillary, high grade & $62(37 \%)$ & NA & \\
Invasive, high grade & $48(28 \%)$ & NA & \\
\hline Abbreviations: NA = not applicable; UC = urothelial carcinoma. &
\end{tabular}

Table 2. Clinical and histopathological characteristics of patients with urothelial carcinoma and of controls (healthy donors $(n=25)$, prostate cancer $(n=25)$ and renal cancer $(n=25)$ patients), which provided urine samples for this study

\begin{tabular}{|c|c|c|c|}
\hline Clinicopathological features & UC & $\begin{array}{c}\text { Control } \\
\text { set }\end{array}$ & $P$-value \\
\hline Patients, $n$ & 49 & 75 & \\
\hline $\begin{array}{l}\text { Gender, n (\%) } \\
\text { Male } \\
\text { Female }\end{array}$ & $\begin{array}{l}29(60 \%) \\
20(40 \%)\end{array}$ & $\begin{array}{l}53(71 \%) \\
22(29 \%)\end{array}$ & \\
\hline Median age, years (range) & $70(53-83)$ & $63(51-88)$ & $P<0.066$ \\
\hline $\begin{array}{l}\text { Grade, } n(\%) \\
\text { Papillary, low grade } \\
\text { Papillary, high grade } \\
\text { Invasive, high grade }\end{array}$ & $\begin{array}{l}17(35 \%) \\
18(37 \%) \\
14(28 \%)\end{array}$ & $\begin{array}{l}\text { NA } \\
\text { NA } \\
\text { NA }\end{array}$ & \\
\hline
\end{tabular}

UTUC, diagnosed and treated between 2006 and 2012 at the Portuguese Oncology Institute - Porto, Portugal. A set of 75 voided urine samples from patients with prostate cancer $(n=25)$, renal cancer $(n=25)$ and healthy blood donors with no personal or familial history of cancer $(n=25)$ were also collected and used as controls (Table 2). Informed consents were obtained from patients and controls and used in this study after approval from the ethics committee (Comissão de Ética para a Saúde) of the Portuguese Oncology Institute of Porto (CES-IPO 019/08). All urine samples were processed by immediate centrifugation at 4000 r.p.m. for $10 \mathrm{~min}$, the respective pellet was washed twice with phosphatebuffered saline and stored at $-80{ }^{\circ} \mathrm{C}$.

Nucleic acids isolation, bisulphite modification and qMSP analysis. DNA was extracted from frozen BUC tissue samples using AllPrep DNA/RNA Mini Kit (Qiagen Inc., Germatown, MD, USA). For UTUC NUTU tissue samples, a representative paraffin block was selected and the tumour area was delimited, allowing for macrodissection of tumour from 10 to 20 serial $7-\mu \mathrm{m}$ thick sections, followed by digestion with proteinase $\mathrm{K}\left(20 \mathrm{mg} \mathrm{ml}^{-1}\right.$, $50 \mu \mathrm{l})$. DNA from all samples was extracted using a standard phenol-chloroform protocol (Pearson and Stirling, 2003), and its concentration determined using ND-1000 NanoDrop (NanoDrop Technologies, Wilmington, DE, USA). Bisulphite modification was performed using sodium bisulphite with EZ DNA MethylationGold Kit (Zymo Research, Irvine, CA, USA) according to manufacturer's protocol. Quantitative methylation levels were 
performed using KAPA SYBR FAST qPCR Kit (Kapa Biosystems, Wilmingtown, MA, USA) and all reactions were run in triplicates in 384-well plates using Roche LightCycler 480 II, with BetaActin $(A C T B)$ as internal reference gene for normalisation. Primer sequences were designed using Methyl Primer Express 1.0 (Methyl Primer Express 1.0, ThermoFisher Scientific, Waltham, MA, USA) and purchased from Sigma-Aldrich (St Louis, MO, USA): miR-129-2 F3'-CGGCGAATCGAAGAAGTC-5' and R3'TACGCCCTCCGCAAATAC-5', miR-663a F3'-GGGATAGCGA GGTTAGGTC-5' and R3'-CATTCGTAACGAATAAAACCC-5'.

Statistical analysis. Median, frequency and interquartile range of miR-129-2 and miR-663a promoter methylation levels of normal, BUC and UTUC tissue samples as well as UC, prostate, kidney and healthy blood donor urine samples were determined. Receiver operator characteristics (ROC) curves were constructed by plotting the true-positive (sensitivity) against false-positive (1-specificity) rate, and the area under the curve (AUC) was calculated. The higher value obtained from the sum of sensitivity and 1-specificity in each ROC curve was used for cut off to categorise samples as methylated or non-methylated. Sensitivity, specificity, negative predictive value, positive predictive value and accuracy of the test were also determined. Differences in quantitative methylation values were assessed with the non-parametric Mann-Whitney $U$ test. Associations between age, gender, grade, pathological stage and miRNAs methylation levels were carried out using Spearman's method, Mann-Whitney or Kruskall-Wallis tests, as appropriate. DeLong's test for ROC curves comparison was performed to assess differences in performance of the miRNAs promoter methylation test between upper and lower urinary tract cancers, and between the age groups (lower than 65 years $v s$ higher than 65 years). McNemar proportion test was used to compare the diagnostic performance of methylation analysis with urine cytology.

Disease-specific survival curves, (Kaplan-Meier with log rank test) were computed for standard variables (tumour stage and grade) and for categorised miRNA promoter methylation status. A Cox regression model comprising all significant variables (univariable and multivariable model) was computed to assess the relative contribution of each variable to the follow-up status.

All two-tailed $P$-values were derived from statistical tests, using a computer-assisted program (SPSS Version 23.0, IBM, Armonk, NY, USA) and the results were considered statistically significant at $P<0.05$. Bonferron is correction for multiple comparisons was used when applicable.

\section{RESULTS}

Methylation analysis in UC tissues and performance of methylation panel in urine. The promoters of both miR-129-2 and miR-663a were found to be methylated in most UC tissue samples, and methylation levels were significantly higher compared with the control group $(P<0.001$ and $P<0.001$, respectively; Figure 1). Moreover, in tissue samples, the panel discriminated UC from normal mucosa with $94.7 \%$ sensitivity and $84.0 \%$ specificity (Table 3), corresponding to an AUC of 0.941 (95\% confidence interval (CI): $0.911-0.972, P<0.001)$ in ROC curve analysis (Figure 2A).

The same panel was then tested in a set of 49 urine sediments from UC patients and in a control group of 75 urines from subjects
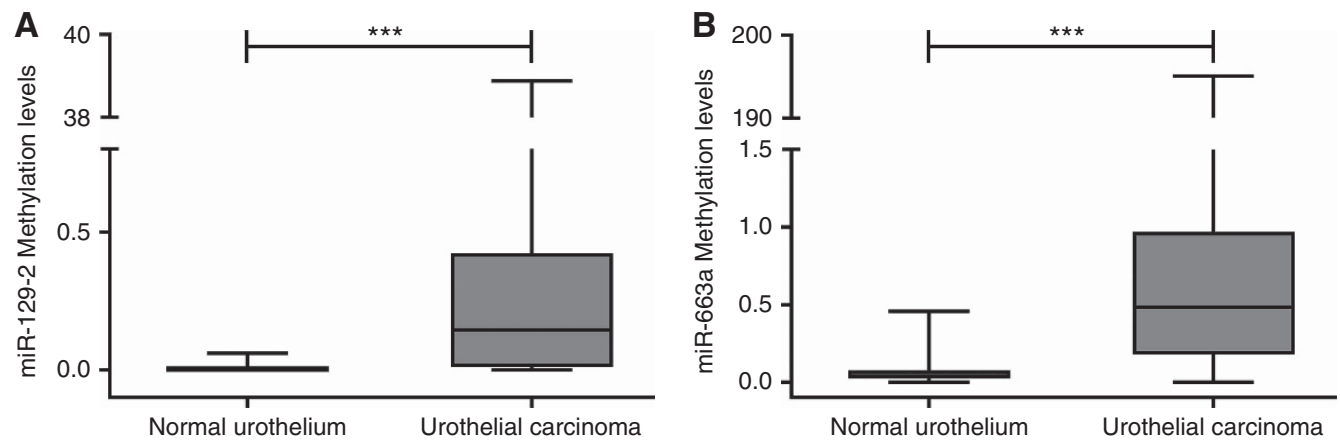

Figure 1. Distribution of (A) miR-129-2 and (B) miR-663a promoter methylation levels in normal urothelium ( $n=50)$ and urothelial carcinoma (UC) tissue samples $(n=169)$. Mann-Whitney $U$ test. ${ }^{\star \star \star} P<0.001$.

\begin{tabular}{|c|c|c|c|c|c|}
\hline & $\begin{array}{c}\text { Sensitivity } \\
\% \text { (n positive/n total) }\end{array}$ & $\begin{array}{c}\text { Specificity } \\
\% \text { (n negative } / \boldsymbol{n} \text { total) }\end{array}$ & PPV (\%) & NPV (\%) & Accuracy (\%) \\
\hline \multicolumn{6}{|l|}{ Tissue samples } \\
\hline miR-129-2 & $72.8(123 / 169)$ & $96.0(48 / 50)$ & 98.4 & 51.1 & 78.1 \\
\hline mirR-663a & $87.0(147 / 169)$ & $86.0(43 / 50)$ & 95.5 & 66.2 & 86.8 \\
\hline miR-129-2/miR-663a & $94.7(160 / 169)$ & $84.0(42 / 50)$ & 95.2 & 82.4 & 92.2 \\
\hline \multicolumn{6}{|c|}{ Urine samples (UC patients vs HD) } \\
\hline miR-129-2/miR-663a & $83.7(41 / 49)$ & $88.0(22 / 25)$ & 93.2 & 73.3 & 85.1 \\
\hline \multicolumn{6}{|c|}{ Urine samples (UC patients vs $\mathrm{PCa}$ and $\mathrm{RC}$ patients) } \\
\hline miR-129-2/miR-663a & $87.8(43 / 49)$ & $84(42 / 50)$ & 84.3 & 87.5 & 85.9 \\
\hline \multicolumn{6}{|c|}{ Urine samples (UC patients vs all controls) } \\
\hline miR-129-2 & $75.5(37 / 49)$ & $85.3(64 / 75)$ & 77.1 & 84.2 & 81.5 \\
\hline mirR-663a & $71.4(35 / 49)$ & $94.7(71 / 75)$ & 89.7 & 83.5 & 85.5 \\
\hline miR-129-2/miR-663a & $87.8(43 / 49)$ & $82.7(62 / 75)$ & 76.8 & 91.2 & 84.7 \\
\hline
\end{tabular}



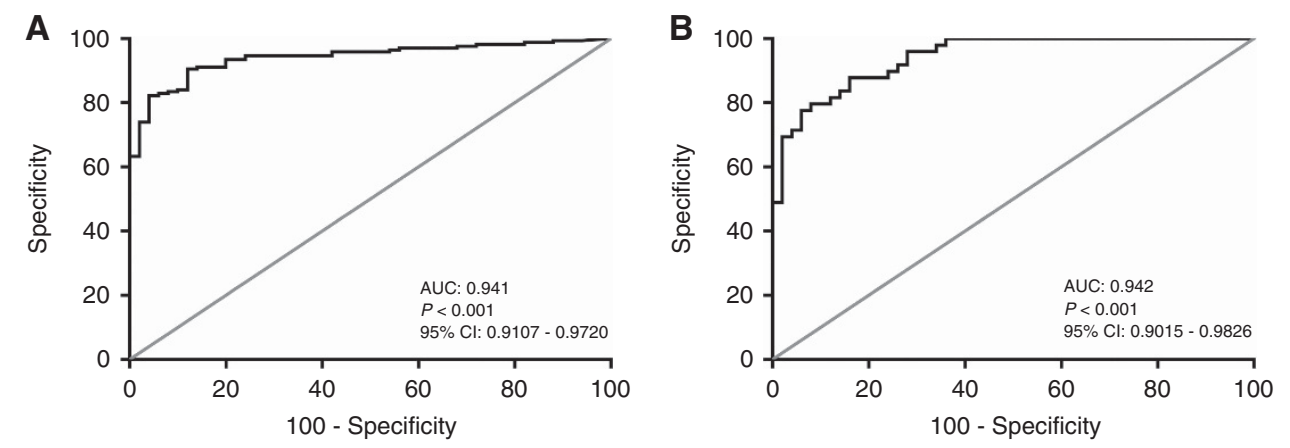

Figure 2. Receiver operator characteristic (ROC) curves evaluating the performance of the gene panel promoter methylation (mir-129-2/miR-663a); (A) for the identification of urothelial carcinoma (UC) in tissue; and (B) for discrimination of UC from other genitourinary malignancies in urine samples.

not carrying UC. Remarkably, methylation levels of both miRNAs in UC urine samples were significantly higher than those of controls $(P<0.001$ and $P<0.001$, respectively). In urine samples, the methylation test was able to detect UC with $87.8 \%$ sensitivity and $84.0 \%$ specificity (Table 3 ), corresponding to an AUC of 0.942 (95\% CI: $0.9015-0.9826, P<0.001$; Figure 2B). Moreover, the methylation test was able to discriminate UC patients both from other genitourinary malignancies and from healthy donors (Table 3).

Because urine cytology is frequently the first test to be performed in UC suspects, we compared the performance of the methylation panel with cytopathological examination by an experienced cytopathologist. Interestingly, the proportion of truepositive cases detected by the methylation test was significantly higher than that of cytology $(P<0.001)$. Of $47 \mathrm{UC}$ cases analysed, cytopathology detected only 17 as positive, 15 as negative and 15 as 'inconclusive/suspicious', corresponding to $34.7 \%$ sensitivity. Conversely, the miRNAs promoter methylation panel identified 41 cases as true positive, corresponding to an overall sensitivity of $87.2 \%$, although 1 of the 6 cases negative in the methylation test was correctly diagnosed as UC by cytopathology (Figure 3 ).

Clinicopathological correlates and survival analyses. Significantly higher miR-129-2 methylation levels were found in highgrade papillary UC compared with low-grade papillary UC $(P=0.048)$, whereas for miR-663a, high-grade papillary UC displayed significantly higher methylation levels than invasive UC $(P=0.003)$. In addition, miR-663a methylation levels differed significantly between non-muscle invasive and muscle invasive UC (stages pTa-1 vs pT2-4; $P=0.016$ ), as well as between papillary and invasive $\mathrm{UC}(P=0.012)$.

A significant association was found between promoter methylation levels and patients' age at diagnosis for both miR-129-2 and miR-663a $(P=0.023 ; P=0.016$, respectively). After normalisation of the ROC curve for this variable, no significant difference in the panel's performance was found between younger and older patients and an AUC of $94.3 \%$ was obtained. Furthermore, no association was disclosed between miRNAs promoter methylation and patients' gender.

Of the 114 patients enrolled, 3 BUC and 1 UTUC patients were lost to follow-up. The median follow-up time of BUC patients was 66 months (range: 1-323 months). At the last follow-up time point, 58 patients were alive with no evidence of cancer, 10 patients were alive with disease, 11 died from other causes and 32 had deceased due to UC. Considering UTUC patients, the follow-up time was of 55 months (range: 1-186 months). At the last follow-up, 16 patients were alive without disease, 6 were alive with disease progression, whereas 32 patients had perished, 23 due to UTUC. Overall, for UC, the median follow-up time was 62 months. A poor

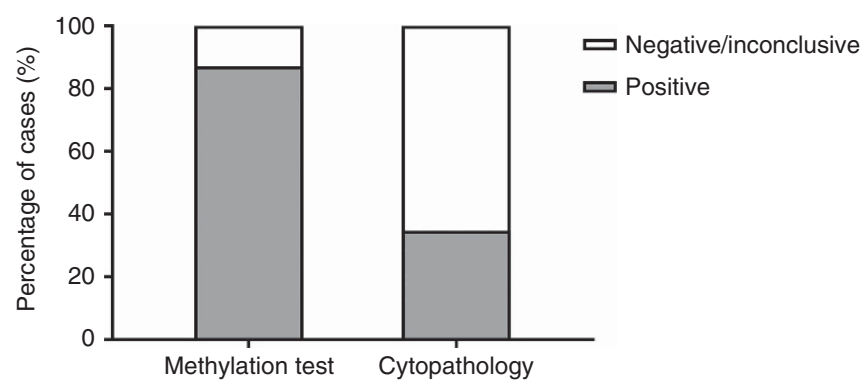

Figure 3. Percentage of urothelial carcinoma (UC) cases correctly identified with the gene panel promoter methylation test and a standard cytopathology analysis.

outcome was depicted for UC patients with higher grade, pathological stage and age at diagnosis (Log rank test; $P<0.001$, for all variables). Univariable and multivariable Cox regression analysis were performed separately for BUC, UTUC and UC patients, including the three above mentioned variables (Supplementary Table 1). As expected, a poor outcome was depicted for UC patients with higher pathological stage, grade and age in a multivariable model (Supplementary Table 1; $P=0.03$, $P=0.002$ and $P<0.001$, respectively). However, considering the two patients' subsets separately, only grade (for BUC) and age (for UTUC) were selected in the final model as independent predictors of outcome (Supplementary Table $1 ; P=0.009$ and $P=0.017$ ). No prognostic value was depicted for miR-129-2 or miR-663a promoter methylation levels in UC or in BUC or UTUC, when analysed separately (Supplementary Table 1).

\section{DISCUSSION}

Upper and lower UC are among the most common neoplasms worldwide and although several risk factors have been clearly identified (e.g., smoking habits, chemical exposure to aromatic amines like benzidine or $\beta$-naphtalene, Schistosoma infection (Babjuk et al, 2013; Torre et al, 2015)), early detection is critical for adequate therapeutic management towards reducing diseasespecific mortality (Hall et al, 1998; Margulis et al, 2009). Moreover, it is important to discriminate UC from other genitourinary cancers, especially those originating in the prostate and kidney. Although several biomarkers have been previously reported, including miRNAs promoter methylation (Phé et al, 2009; van der Kwast and Bapat, 2009; Shimizu et al, 2013), they have been mostly focused on BUC, disregarding upper urothelial tract UC, and its performance might be perfected by the addition of more 
sensitive and specific biomarkers. Within a project aimed at characterising miRNAs deregulated through aberrant promoter methylation in genitourinary neoplasms, we identified miR-129-2 and miR-663a promoters as potential UC biomarkers (submitted). We, thus, tested the biomarker performance of quantitative miR-129-2 and miR-663a promoter methylation both in upper and lower urinary tract UC.

Because miR-129-2 and miR-663a promoter methylation was initially identified in BUC, we first assessed methylation levels in tissue samples of upper and lower urinary tract UC. Owing to biological and genomic similarity between the urothelium from upper and lower urinary tract (Zhang et al, 2010), we hypothesised that this panel would perform well in both the settings. Indeed, the methylation panel discriminated UC from normal urothelial mucosa with high sensitivity and specificity, which did not differ between upper and lower urinary tract UC. This result enabled us to proceed with urine testing, as the ultimate goal of the study would be the identification of a non-invasive test, intended for early detection and disease monitoring. In urine samples, sensitivity and specificity were lower than those found in tissues, but it should be recalled that the accuracy of the panel was tested not only against healthy volunteers, but also prostate and kidney cancer patients.

Recently, several studies attempted to identify novel epigenetic biomarkers for UC detection, some of them with an apparent superior performance to the panel reported herein. TWIST1 and NID2 promoter methylation were previously reported to detect BUC in urine samples with $94 \%$ sensitivity and $91 \%$ specificity (Renard et al, 2010). However, specificity was only tested against urinary infections or other benign conditions and its ability to discriminate UC from prostate and kidney cancer was not evaluated. BCL2, CDKN2A and NID2 promoter methylation have also been proposed as epigenetic biomarkers for bladder cancer (Scher et al, 2012). Although the number of genes is higher than that of our panel, sensitivity and specificity were lower $(80.9 \%$ and $86.4 \%$, respectively) and this was accomplished through nested PCR, which may compromise the speed and cost of the assay. Moreover, the number of samples from prostate and kidney cancer tested was lower than those included in our study. We have also previously reported a gene promoter methylation panel (GDF15, TMEFF2 and VIM) that accurately identified BUC in urine samples (Costa et al, 2010) which we, subsequently, demonstrated to have similar performance in upper urinary tract UC (MonteiroReis et al, 2014). Both studies, however, used specific TaqMan probes, contrarily to the present study, where a SYBR Green-based protocol was used, thus, also, improving cost-effectiveness.

Some previous studies have also focused on miRNAs promoter methylation as UC biomarkers. Whereas, Vogt et al (2011) reported $57 \%$ sensitivity $(n=7)$ for miR-34a promoter methylation in bladder tissues and Shimizu et al (2013) achieved $81 \%$ specificity and $89 \%$ sensitivity in urine sediments from BUC $(n=47)$ using a panel of several miRNAs. Our results compare well with those reports and provide some significant advantages, as only two miRNA promoters are tested and its specificity was evaluated against other genitourinary malignancies.

Although urinary cytology is frequently used as an initial diagnostic approach in UC suspects, its diagnostic yield is rather limited, especially for upper urinary tract UC (Rouprêt et al, 2013). Moreover, imaging techniques might have difficulty in discriminating upper urinary tract UC from renal cell carcinoma, a quite relevant differential diagnosis setting owing to marked differences in therapy and prognosis (Browne et al, 2005), thus emphasising the need for biomarkers that may accurately discriminate among those tumour types. In the present study, the sensitivity of urinary cytology was only $\sim 35 \%$, which was easily surpassed by the miRNA methylation panel, with the additional gain of discriminating UC from renal cell carcinoma.
Whereas no biological role has been previously ascribed to miR-129-2 and miR-663a promoter methylation in urothelial carcinogenesis, several studies in other tumour models have unveiled the pathological significance of those epigenetic aberrations. Transcriptional silencing of miR-129-2 due to promoter methylation was found in gastric (Pan et al, 2010; Shen et al, 2010), endometrial (Huang et al, 2009) and hepatocellular (Lu et al, 2013) carcinomas, as well as in acute myeloid leukaemia (Yan-Fang et al, 2013), and it has been implicated in overexpression of two oncogenic proteins, SOX4 (Huang et al, 2009; Shen et al, 2010) and Cdk6 (Wu et al, 2010). On the other hand, miR-663a promoter methylation and downregulation was associated with JunD overexpression in small-cell lung carcinoma (Zhang et al, 2016) and HMGA2 overexpression in hepatocellular carcinoma (Huang et al, 2016), fostering cell proliferation. Owing to the prevalence of miR129-2 and miR-663a promoter methylation in UC, across primary localisations, histological subtype, grade and stage, it is tempting to speculate whether it may also play a key role in urothelial carcinogenesis.

In summary, we demonstrated that aberrant miR-129-2 and miR-663a promoter methylation accurately discriminate UC from normal urothelial mucosa and allow for sensitive and specific identification of upper and lower urinary tract UC in urine samples, discriminating also from other common genitourinary tract carcinomas (kidney and prostate). Thus, this panel might be useful for complementing other epigenetic biomarkers for noninvasive detection and/or monitoring of UC patients.

\section{ACKNOWLEDGEMENTS}

We are grateful to $\mathrm{M}^{\mathrm{a}}$ - Conceição Martins, BSc for the skilful technical support and would like to acknowledge the nursing staff of the Departments of Urology and Laboratory Medicine of the Portuguese Oncology Institute of Porto for their collaboration in urine collection. This study was supported by research grants from Research Center of Portuguese Oncology Institute of Porto (CIIPOP-FBGEBC-27) and by Federal funds through Programa Operacional Temático Factores de Competitividade (COMPETE) with co-participation from the European Community Fund (FEDER) and by national funds through Fundação para a Ciência e Tecnologia (FCT) under the projects EXPL/BIM-ONC/0556/ 2012. SM-R and JR-C are PhD students funded by FCT-Fundação para a Ciência e a Tecnologia fellowship (SFRH/BD/112673/2015 and SFRH/BD/71293/2010, respectively).

\section{CONFLICT OF INTEREST}

The authors declare no conflict of interest.

\section{AUTHOR CONTRIBUTIONS}

Conceived and designed the experiments: RH, CJ. Performed the experiments: NAP, SM-R, JT-F, LL, DM, JR-C, PCD, PM and JO. Data analysis and interpretation: NAP, SM-R, LA, RH and CJ. Contributed reagents/material/analysis: JT-F, PM, JO, RH and CJ; Manuscript preparation: NAP, SM-R, RH and CJ.

\section{REFERENCES}

Ambros V (2004) The functions of animal microRNAs. Nature 431(7006): 350-355.

Babjuk M, Burger M, Zigeuner R, Shariat SF, van Rhijn BW, Comperat E, Sylvester RJ, Kaasinen E, Böhle A, Redorta JP (2013) EAU guidelines on 
non-muscle-invasive urothelial carcinoma of the bladder: update 2013. Eur Urol 64(4): 639-653.

Bartel DP (2009) MicroRNAs: target recognition and regulatory functions. Cell 136(2): 215-233.

Browne RF, Meehan CP, Colville J, Power R, Torreggiani WC (2005) Transitional cell carcinoma of the upper urinary tract: spectrum of imaging findings 1. Radiographics 25(6): 1609-1627.

Costa VL, Henrique R, Danielsen SA, Duarte-Pereira S, Eknaes M, Skotheim RI, Rodrigues Â, Magalhães JS, Oliveira J, Lothe RA (2010) Three epigenetic biomarkers, GDF15, TMEFF2, and VIM, accurately predict bladder cancer from DNA-based analyses of urine samples. Clin Cancer Res 16(23): 5842-5851.

Eble J, Sauter G, Epstein J, Sesterhenn I (2004) Tumours of the urinary system and male genital organs: pathology and genetics. World Health Organization Classification of Tumours. IARC Press: Lyon.

Edge SB BD, Compton CC, Fritz AG, Greene FL, Trotti A (2010) American Joint Committee on Cancer: Cancer Staging Manual. 7th edn. Lippincott-Raven Publishers: Philadelphia.

Hall MC, Womack S, Sagalowsky AI, Carmody T, Erickstad MD, Roehrborn CG (1998) Prognostic factors, recurrence, and survival in transitional cell carcinoma of the upper urinary tract: a 30-year experience in 252 patients. Urology 52(4): 594-601.

Huang W, Li J, Guo X, Zhao Y, Yuan X (2016) miR-663a inhibits hepatocellular carcinoma cell proliferation and invasion by targeting HMGA2. Biomed Pharmacother 81: 431-438.

Huang Y-W, Liu JC, Deatherage DE, Luo J, Mutch DG, Goodfellow PJ, Miller DS, Huang TH (2009) Epigenetic repression of microRNA-129-2 leads to overexpression of SOX4 oncogene in endometrial cancer. Cancer Res 69(23): 9038-9046.

Kaufman DS, Shipley WU, Feldman AS (2009) Bladder cancer. Lancet 374(9685): 239-249.

Lokeshwar VB, Habuchi T, Grossman HB, Murphy WM, Hautmann SH, Hemstreet GP, Bono AV, Getzenberg RH, Goebell P, Schmitz-Dräger BJ (2005) Bladder tumor markers beyond cytology: international Consensus Panel on bladder tumor markers. Urology 66(6): 35-63.

Lu CY, Lin KY, Tien MT, Wu CT, Uen YH, Tseng TL (2013) Frequent DNA methylation of MiR-129-2 and its potential clinical implication in hepatocellular carcinoma. Genes Chromosomes Cancer 52(7): 636-643.

Margulis V, Shariat SF, Matin SF, Kamat AM, Zigeuner R, Kikuchi E, Lotan Y, Weizer A, Raman JD, Wood CG (2009) Outcomes of radical nephroureterectomy: a series from the Upper Tract Urothelial Carcinoma Collaboration. Cancer 115(6): 1224-1233.

Monteiro-Reis S, Leça L, Almeida M, Antunes L, Monteiro P, Dias PC, Morais A, Oliveira J, Henrique R, Jerónimo C (2014) Accurate detection of upper tract urothelial carcinoma in tissue and urine by means of quantitative GDF15, TMEFF2 and VIM promoter methylation. Eur J Cancer 50(1): 226-233.

Pan J, Hu H, Zhou Z, Sun L, Peng L, Yu L, Sun L, Liu J, Yang Z, Ran Y (2010) Tumor-suppressive mir-663 gene induces mitotic catastrophe growth arrest in human gastric cancer cells. Oncol Rep 24(1): 105-112.

Pearson H, Stirling D (2003) DNA extraction from tissue. Methods Mol Biol 226: 33-34.

Phé V, Cussenot O, Rouprêt M (2009) Interest of methylated genes as biomarkers in urothelial cell carcinomas of the urinary tract. BJU Int 104(7): 896-901.

Remzi M, Shariat S, Huebner W, Fajkovic H, Seitz C (2011) Upper urinary tract urothelial carcinoma: what have we learned in the last 4 years? Ther Adv Urol 3(2): 69-80.

Renard I, Joniau S, Van Cleynenbreugel B, Collette C, Naômé C, Vlassenbroeck I, Nicolas H, de Leval J, Straub J, Van Criekinge W (2010)
Identification and validation of the methylated TWIST1 and NID2 genes through real-time methylation-specific polymerase chain reaction assays for the noninvasive detection of primary bladder cancer in urine samples. Eur Urol 58(1): 96-104.

Rouprêt M, Babjuk M, Compérat E, Zigeuner R, Sylvester R, Burger M, Cowan N, Böhle A, Van Rhijn BW, Kaasinen E (2013) European guidelines on upper tract urothelial carcinomas: 2013 update. Eur Urol 63(6): 1059-1071.

Rouprêt M, Zigeuner R, Palou J, Boehle A, Kaasinen E, Sylvester R, Babjuk M, Oosterlinck W (2011) European guidelines for the diagnosis and management of upper urinary tract urothelial cell carcinomas: 2011 update. Eur Urol 59(4): 584-594.

Scher MB, Elbaum MB, Mogilevkin Y, Hilbert DW, Mydlo JH, Sidi AA, Adelson ME, Mordechai E, Trama JP (2012) Detecting DNA methylation of the BCL2, CDKN2A and NID2 genes in urine using a nested methylation specific polymerase chain reaction assay to predict bladder cancer. J Urol 188(6): 2101-2107.

Shen R, Pan S, Qi S, Lin X, Cheng S (2010) Epigenetic repression of microRNA-129-2 leads to overexpression of SOX4 in gastric cancer. Biochem Biophys Res Commun 394(4): 1047-1052.

Shimizu T, Suzuki H, Nojima M, Kitamura H, Yamamoto E, Maruyama R, Ashida M, Hatahira T, Kai M, Masumori N (2013) Methylation of a panel of microRNA genes is a novel biomarker for detection of bladder cancer. Eur Urol 63(6): 1091-1100.

Silahtaroglu A, Stenvang J (2010) MicroRNAs, epigenetics and disease. Essays Biochem 48: 165-185.

Torre LA, Bray F, Siegel RL, Ferlay J, Lortet-Tieulent J, Jemal A (2015) Global cancer statistics, 2012. CA Cancer J Clin 65(2): 87-108.

van der Kwast TH, Bapat B (2009) Predicting favourable prognosis of urothelial carcinoma: gene expression and genome profiling. Curr Opin Urol 19(5): 516-521.

Vogt M, Munding J, Grüner M, Liffers S-T, Verdoodt B, Hauk J, Steinstraesser L, Tannapfel A, Hermeking H (2011) Frequent concomitant inactivation of miR-34a and miR-34b/c by CpG methylation in colorectal, pancreatic, mammary, ovarian, urothelial, and renal cell carcinomas and soft tissue sarcomas. Virchows Arch 458(3): 313-322.

Volinia S, Calin GA, Liu C-G, Ambs S, Cimmino A, Petrocca F, Visone R, Iorio M, Roldo C, Ferracin M (2006) A microRNA expression signature of human solid tumors defines cancer gene targets. Proc Natl Acad Sci USA 103(7): 2257-2261.

Wu J, Qian J, Li C, Kwok L, Cheng F, Liu P, Perdomo C, Kotton D, Vaziri C, Anderlind C (2010) miR-129 regulates cell proliferation by downregulating Cdk6 expression. Cell Cycle 9(9): 1809-1818.

Yan-Fang T, Jian N, Jun L, Na W, Pei-Fang X, Wen-Li Z, Dong W, Li P, Jian W, Xing F (2013) The promoter of miR-663 is hypermethylated in Chinese pediatric acute myeloid leukemia (AML). BMC Med Genet 14(1): 74 .

Zhang Y, Xu X, Zhang M, Wang X, Bai X, Li H, Kan L, Zhou Y, Niu H, He P (2016) MicroRNA-663a is downregulated in non-small cell lung cancer and inhibits proliferation and invasion by targeting JunD. BMC Cancer 16(1): 1.

Zhang Z, Furge KA, Yang XJ, Teh BT, Hansel DE (2010) Comparative gene expression profiling analysis of urothelial carcinoma of the renal pelvis and bladder. BMC Med Genomics 3(1): 58.

This work is published under the standard license to publish agreement. After 12 months the work will become freely available and the license terms will switch to a Creative Commons AttributionNonCommercial-Share Alike 4.0 Unported License.

Supplementary Information accompanies this paper on British Journal of Cancer website (http://www.nature.com/bjc) 